\title{
Morphogenesis-Based Deformable Models Application to 3D Medical Image Segmentation and Analysis
}

\author{
Luis Ibáñez ${ }^{1}$, Chafiaâ Hamitouche ${ }^{2}$, Martial Boniou ${ }^{2}$, and Christian Roux ${ }^{2}$ \\ 1 Computer science department, division of neurosurgery,UNC, USA \\ 2 Département ITI - LATIM -INSERM 0102, ENST Bretagne, France \\ chafiaa.hamitouche@enst-bretagne.fr
}

\begin{abstract}
This paper introduces the concept of structured deformable model and presents its application to 3D medical image analysis. A structured deformable model is composed of a group of basic shape elements. A generic model of a biological shape can be build following a growth process controlled by the morphogenesis. This model can be fitted to a specific biological shape by adjusting the parameters of the model based on the information given by a 3D image of the targeted biological structure. An application to bone joint modelling is presented and discussed.
\end{abstract}

\section{Introduction}

Since the introduction of Snakes [1], Deformable models have been widely used in applications related with image segmentation of biological shapes [2]. The approach presented in this paper follows the trend of introducing more anatomical information and specific behavior into models. It designs a deformable model by considering the biological process responsible of organ morphogenesis. The hypothesis is that we can better model a form if we take into account the real biological mechanisms that leads to shape formation.

\section{Modelling and Segmentation of Osteo-articular Shapes}

Morphogenesis Knowledge Biological shape is the result of dynamical systems evolution [3]. Organ shape is, in fact, the spatial distribution of a particular kind of cells and a specific inter-cellular material. Biological shape results from interactions of tissues at cellular level. A tissue finish in space when particular conditions needed to maintain its coherence falls under a certain threshold. These considerations lead to the hypothesis that the families of shapes found in biological organs should belong to the varieties of shapes of zero sets found in implicit functions. In the case of bone modelling, the model is designed by considering the relative positions and sizes of chondrification centers and the geometric configurations of tendons and muscles insertions, using implicit surfaces to emulate the local morphology as described in osteology, arthrology and myology. 
Building Generic Shapes. Application to the Elbow Joint Metaballs paradigm was chosen here to build our model by making a gaussian sum of implicit functions that represent our ossification centers like local morphogenetic fields controlled by a few parameters. The articulation to model is composed of three bones epiphyses : the distal region of humerus, the radius head, and the ulna. An homotopy process 4 for mesh constructing implies a progressive modelling from an initial state where each element is collapsed into a simple shape (sphere) with a known mesh for each of the three bone extremities. Then the parameters variations imply a simulated morphogenesis until an adult final stage. The elbow modelling is shown in Figure 1. In order to fit the structured model against the real image, a good rigid transformation is performed to superimpose one over the other in an optimization process.
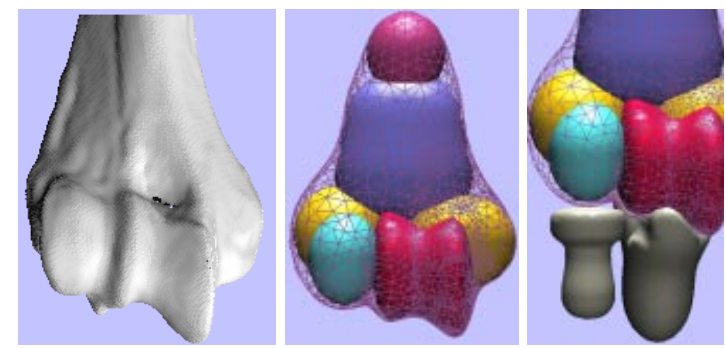

Fig. 1. Humerus distal head (left). Model (center). Complete Elbow Model (right).

\section{Conclusion}

The structured deformable model presented in this paper is a flexible tool for modelling biological shapes. The main advantage of this method is the possibility of interpreting in biological terms all the elements composing the model. Future work will be directed to the analysis of particular shapes with the aim of determining the appropriated combination of basic metaball elements that should be used to reproduce a good approximation of the shape.

\section{References}

1. M. Kass, A. Witkin and D. Terzopoulos, "Snakes: Active contour models", Int. J. Comput. Vision, Vol. 1, No. 4, 1988, 321-331.

2. T. McInerney, D. Terzopoulos, "Deformable models in medical image analysis", Proc. Workshop on Math. Methods in Biomed. Image Anal., June 1996, 171 - 180.

3. R. Thom, Stabilité Structurelle et Morphogénèse, Essai d'une thoèrie générale des modèles. ed. InterEditions, 2nd Ed. 1977.

4. H. Lamure, D. Michelucci, Solving Geometric Constraints By Homotopy, IEEE TVCG, Vol 2, N. 1, Mar. 1996, 28-35. 